\title{
Salmonella and rivals PREEMPTed from chickens
}

A product containing a defined consortium of 29 types of live bacteria has been approved by the US Food and Drug Administration (FDA; Rockville, MD) to prevent Salmonella infections in chickens. It will probably be launched in May, which could mean that unapproved competing products will be driven from the US market. PREEMPT, marketed by MS Bioscience (Dundee, IL), but developed by researchers at the USDA's Food Animal Protection Research Laboratory (College Station, TX), has been field tested on 80,000 chickens; not one of the treated chickens became colonized with Salmonella; in contrast, Salmonella was present in about $7 \%$ of the untreated birds. Similar products are in development for reducing infections in pork and beef products.

PREEMPT is sprayed as a mist over newly hatched chicks. Whereas chickens that are at least 3 weeks old have a natural resistance to Salmonella colonization because the gut is already colonized by $300-400$ different commensal bacteria, young chicks lack this protective flora. When PREEMPT (which contains a mixture of commensal intestinal bacteria isolated from adult birds) is sprayed over newborn chicks, it helps establish competitive exclusion-where commensal flora flourish and exclude pathogens-in the naive intestinal tract. The newly hatched chicks ingest the water-based bacterial soup as they groom their feathers.

Competitive exclusion is not novel in concept. PREEMPT will have to compete with two other competitive exclusion products sold in the US: Broilac, made by Orion (Espoo, Finland), which has been in use in Europe since the late 1980s, and Avaguard from Bayer (Leverkusen, Germany). Neither of these has FDA approval, however. "The FDA took a nonenforcement approach to these undefined products," says MS BioScience's regulatory manager, Liz Dunlap. As long as they were viewed as safe, and no good alternative was available, their sale was not contested. MS Bioscience now expects the FDA to enforce regulations against the sale of these products in the United States. It is the defined nature of PREEMPT that gives the product an edge, the company says.

In the original work that led to the product, the USDA researchers set up a continuous flow culture system that mimicked the environment in the adult avian cecum. The two cecal pouches, found at the juncture of

Vicki Glaser is a freelance writer working in Allentown, PA the small intestine and the colon in chickens, are the reservoirs for Salmonella colonization. "If you can prevent Salmonella from getting into the ceca, they won't survive in the rest of the gastrointestinal tract, and will get washed out with normal peristalsis," says project leader Donald Corrier.

The best of the experimental culture systems grew a stable mixture of 29 bacteria when the chemostat reached equilibrium, and that culture proved to be highly protec-

\section{IMAGE UNAVAILABLE FOR COPYRIGHT REASONS}

PREEMPT helps establish competitive exclusion in the naive intestinal tract, offering an alternative to the use of antibiotics in the fight against Salmonella.

tive in chicks. The number of Salmonella per gram of cecal content dropped from $1,000,000$ per gram to less than 10 per gram in treated chicks.

By identifying each bacterial component of the culture and demonstrating the absence of Mycoplasma, viruses, or other potentially harmful components, the researchers were able to present a defined culture to the FDA, which classifies these types of cultures as drugs, and requires proof of safety and efficacy.

The mechanisms of competitive exclusion are not understood. Cultures containing just a few types of organisms have not been effective, and establishing a culture environment able to sustain a large mixture of bacteria has posed a challenge. In fact, some of the bacteria present in the 29-bug culture may not contribute directly to the competitive exclusion mechanism, but may produce a fermentation substrate essential for the growth of other bacteria in the culture.

The sizable US market for PREEMPT includes the 9 billion broiler chickens hatched each year and the approximately 80 million multiplier breeder birds that produce eggs. Even though the product offers an alternative to the use of large doses of antibiotics, acceptance could be an issue: "The broiler industry is on a tight margin and doesn't like anything that adds cost," remarks Dunlap.
Eighteen months ago, the USDA began applying its competitive exclusion strategy to swine. "A culture has been developed, it has been defined, and the patent is pending," according to Corrier. The technique is proving effective in preventing the spread of Salmonella in pigs.

Next on the menu is the prevention of Escherichia coli 0157:H7 in cattle. During the marketing process, the feed is withdrawn for the 24 hours before slaughter, causing a dramatic change in the rumen components: the $\mathrm{pH}$ increases, and the volatile fatty acids normally present in high levels in the rumen due to the fermentation activity of normal flora decrease. As a result, "You get a bloom of E. coli 0157:H7," notes Corrier.

The potential for using a defined human gut culture has attracted attention as well from the pharmaceutical industry. Longterm antibiotic treatment and many forms of chemotherapy, for example, can wipe out the normal intestinal flora. Corrier sees a role for human type cultures "to replenish and keep the normal good guys going."

\section{Vicki Glaser}

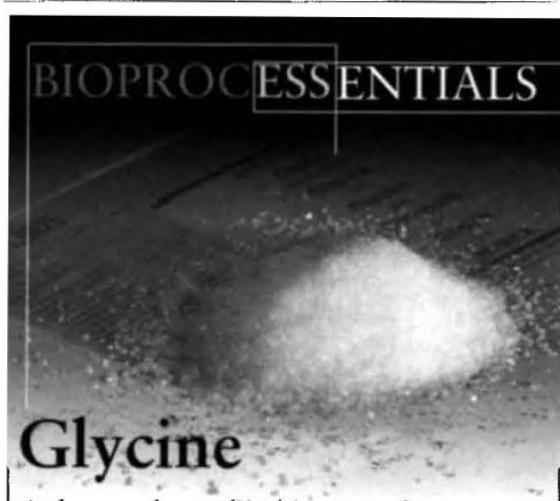

is frequently used in bioprocessing, e.g. as:

- A buffer

- A component of fermentation and cell culture media

- For the stabilisation of protein preparations.

When using glycine and other amino acids from Merck KGaA, you will always be on the safe side; only selected raw materials, essentially of non-animal origin, are used in the fermentation and synthetic production processes involved. The amino acids produced in this way not only fulfil the requirements of all important pharmacopoeias (USP, DAB, $\mathrm{Ph}$ Eur, $\mathrm{BP}$ ) but in fact exceed them in many of the specifications.

Merck KGaA Darmstadt · Germany 